\title{
ROLE OF VITAMIN D3 LEVELS IN PROGNOSTICATION OF PATIENTS DIAGNOSED OF HODGKIN'S LYMPHOMA: A PROSPECTIVE COHORT STUDY
}

\author{
Dr. Akshay Sharma MD Medicine, Medical officer, Civil hospital Baijnath, kangra (HP). \\ Dr. Abhimanyu \\ Patial*
}

\section{ABSTRACT}

Hodgkin's lymphoma (HL) constitutes around 30\% of all lymphomas . IPS is used for the risk stratification of advanced stage HL but it lack applicability to the early stage cases ..In this prospective cohort study, we aim to confirm the relationship between vitaminD3 levels with treatment response (interim as well as end of treatment) of HL treated with the standard chemotherapy and radiotherapy regimens. A total of 50 patients were recruited over a period of 18 months and the patients were followed up in the hematology clinic and interim treatment responses as well as end of treatment response were assessed. Correlation of the vitaminD3 levels and the treatment response showed that the low levels of vitaminD3 were associated with more advanced stages. Univariate analysis showed that there was no significant correlation of interim treatment response as well as end of treatment response with vitamin D3 levels.

\section{KEYWORDS :}

\section{INTRODUCTION}

Hodgkin's lymphoma (HL) constitutes around $30 \%$ of all lymphomas(1). HL is divided into two major categories:

1. Nodular Lymphocyte predominant Hodgkin's lymphoma (NLPHL)

2. Classical Hodgkin's lymphoma (cHL)

cHL represent nearly $95 \%$ of all the cases of HL(2).A cure can be achieved in approximately $80 \%$ of the patients with current treatment regimen consisting of multi agent chemotherapy and radiotherapy (in selected cases).The remaining 20\% attaining remission(4).Early identification of the patients who would fail treatment or relapse in future is currently a challenge. At present the most commonly used tool for prognostication of HL cases is international prognostic score(IPS) (on a scale of 0 -7), with higher scores indicating poorer prognosis)(5).This score is a combination of clinical and basic haematological parameters recorded at the time of diagnosis of a case like age, gender, stage, haemoglobin level, albumin level and lymphocyte counts (1). Since very few types of food naturally contain vitamin $\mathrm{D}$, solar UVB radiation is the main source of vitamin D3 for humans During exposure to solar radiation, 7-dehydrocholesterol (provitamin D3) in epidermis and dermis absorbs UVB radiation and is converted to previtamin D3, which, in turn, is isomerised in the skin to vitamin D3(3). Once formed in the skin, vitamin D3 enters the circulation and is metabolised in the liver by 25 hydroxylase to 25-hydroxyvitamin D3 (calcidiol), which is further converted in the kidney by lahydroxylase to la,25dihyroxyvitamin D3 (calcitriol)(3). This active metabolite binds to the nuclear receptors in the intestine, bone and kidney to perform the calcaemic function in the bone and mineral metabolism (Ultraviolet B radiation from the sun contributes strongly to the vitamin D status in humans. Norway (581-711N) has pronounced seasonal variation in the UV fluence rate. During the summer season, the UV radiation is moderately strong, while during the winter season, practically no vitamin $D$ is generated by UV radiation(6). The maximal concentration of calcidiol (25-hydroxyvitamin D3), which has been found in July-September , is about $50 \%$ higher than the baseline level measured during winter, and differentiation of cancer cells and the serum level of the vitamin D metabolite calcidiol is almost $50 \%$ higher in the summer than in the winter season. Similarly, using cancer registry data in Norway during 1964-2000,Porojnicu et al. [29] reported a 20\% lower fatality rate for patients with Hodgkin's lymphoma diagnosed in autumn versus those diagnosed in winter(6).This association was stronger (60\% lower mortality) among patients younger than 30 years old at the time of diagnosis. In this study we aim to study the role of vitamin $\mathrm{D}$ to prognosticate the patient of Hodgkin's lymphoma(6).

\section{MATERIAL AND METHODS}

Study Design

This was a prospective cohort study carried out in the patients diagnosed with Hodgkin's lymphoma who completed treatment at PGIMER, Chandigarh. Patients were enrolled in the study from the Adult Haematology Clinic PGIMER, Chandigarh prospectively from July 2017 to March 2018 and then they were followed up till November 2018.

Duration Ofthe Study

July2017-December. 2018

Place Of The Study:

1.Department of Internal Medicine Adult Clinical Haematology Unit PGIMER, Chandigarh

2. Department of Histopathology, PGIMER, Chandigarh

\section{Inclusion Criteria}

1.Primary diagnosis of Hodgkin's lymphoma confirmed by a tissue biopsy

2. Adequate lymph node tissue in the paraffin block

3. Treatment naive patients

\section{Exclusion Criteria}

1. Patient previously treated with chemo radiotherapy before coming to PGIMER, Chandigarh.

2. Patients who did not complete chemotherapy or end of treatment chemotherapy response assessment were excluded from further analysis.

\section{METHODOLOGY}

\section{Procedure}

1. Demographic and disease data were recorded in a predesigned performa (annexure 3) and investigations were recorded as per the performa.

2. Collection of the clinical and the laboratory data of the newly enrolled cases was done in the study performa.

3. Biopsy numbers of the lymph node were taken from the Adult Haematology Clinic case record files and the paraffin block were retrieved from the archive of the Department of Histopathology.

4. Cases with adequate tissue in the paraffin block in the archives of the Department of Histopathology were selected and immunohistochemistry staining was performed.

5. Vitamin D3 levels were done before starting the treatment 
of the patients fulfilling the inclusion criteria.

6. Correlation of vitamin D3 levels, and treatment response ( interim as well as end of treatment response was assessed.

7. The interim treatment response was assessed with the PET CT SCAN after two cycles of the chemotherapy and the response was assessed by the Deauville criteria.

8. The end of treatment response was assessed with the PETSCAN after completion of chemotherapy and the response was assessed by Deauville criteria.

\section{STATISTICAL ANALYSIS}

The descriptive statistics were used to study the response rates,. Correlation was assessed between vitamin D3 levels and the response rate by using Chi square test. .Differences were considered as significant if the computed $p$ value was less than theoretical $p$ value i.e. 0.05 . Univariate analysis was performed to assess the association between the vitaminD3 levels with the treatment response with SPSS, version 22.0.

\section{RESULTS}

During the study period, we recruited 60 consecutive, treatment naive patients of Hodgkin's lymphoma. Out of this cohort, there were few exclusions as following:

A. Three patients died before starting treatment.

B. Seven patients lost to follow up before completion of therapy.

Hence, their response $\sim$ to chemotherapy was not available (due to leaving the treatment before completion). Therefore, in the end, total 52 patients were selected. The patients were followed up in the hematology clinic and interim treatment responses as well as end of treatment response were assessed.

\section{VITAMIN D3 LEVELS(25(OH)D $)_{3}$ LEVELS:}

Vitamin $\mathrm{D}_{3}\left(25(\mathrm{OH}) \mathrm{D}_{3}\right)$ levels were estimated in the patients under our study with cut off value of $20 \mathrm{ng} / \mathrm{ml}$, it was found that $53.8 \%$ of the patients were having cut off value less than $20 \mathrm{ng} / \mathrm{ml}$ while $46.8 \%$ of the patients having cut off value greater than $20 \mathrm{ng} / \mathrm{ml}$. Mean absolute value was $22 \mathrm{ng} / \mathrm{ml}$ .Advanced stages of disease were associated with lower vitamin $\mathrm{D}_{3}$ levels as compared to early stages disease. There was no significant correlation ( $\mathrm{p}$ value 0.15 ) found between vitamin $\mathrm{D}_{3}$ levels and interim as well as end of treatment response.

\section{ANALYSIS:}

Interim Treatment Response:

Univariate analysis showed that total of 39 patients $21(53.8 \%)$ had vitamin D3 levels $<20$ and $18(46 \%)$ had vitaminD3 levels $>20$, similarly a total of 9 patients not in complete remission $5(55.5 \%)$ had vitamin D3 levels $<20$ and 4 (44.4\%) had vitamin D3 levels $>20$ Also,there was no significant correlation of interim treatment response with vitaminD3 as shown in table nol.

\section{End Of Treatment Response:}

Univariate analysis showed that total of 48 patients in complete remission $25(52 \%)$ had vitaminD3 levels $<20$ and $23(48 \%)$ had vitaminD3 levels $>20$, similarly a total of 4 patients not in complete remission $3(75 \%)$ had vitaminD3 levels $<20$ and 1 patient (25\%) had vitamin D3 levles $>20$. Also, there was no significant correlation of end of treatment response with vitaminD3 levels as shown in table no 2 .

\section{DISCUSSION}

In this study, which is the prospective investigation carried out over a period of 18 months based on data available, we observed no association between circulating 25(OH)D concentration and overall risk of Hodgkin's lymphoma. Our findings do not support a protective effect of increasing prediagnostic 25(OH)D concentrations on HL risk. The negative findings are largely consistent with reports from other prospective studies $(18,19)$. The most recent published data from the Cohort Consortium Vitamin D Pooling Project similarly showed no decrease in lymphoma risk in relation to elevated concentrations of 25(OH)D overall ( $n=1353$ ) or with common HL subtypes (19).. Previous study has reported that vitamin D receptor is strongly expressed in tumor cells of HL (22). In the present study, vitamin $D_{3}$ level in advanced stages of HL was found to be lower than in early stages. However we do not found any significant correlation between vitamin $\mathrm{D}_{3}$ levels at early and advanced stages. Present study showed that there was no significant correlation of remission at interim as well as at the end of treatment with vitaminD3 levels. However, more evidences would be required by further research, vitaminD3 levels could be developed as a clinical tool for the assessment of the end of treatment response in patients with HL.

In the present study, we intended to correlate vitaminD3 levels with treatment response and survival outcomes but due to short follow up period of our study with only 4 relapses and no mortality, the effective number of events were very less. Hence we limited our study end point to treatment response only. Although present study put forward some evidence which bridge the gap of existing knowledge about role of $\mathrm{Ml}$ and M2 tumor related macrophages phenotype in treatment response in patients with $\mathrm{HL}$, there still exist some limitation in present study.

\section{CONCLUSION}

In conclusion, findings from the current investigation together with the results from previous prospective studies do not provide support between plasma 25(OH)D and Hodgkin's lymphoma risk. However, our data suggest that and low levels of vitamin $\mathrm{D}_{3}$ were associated with advanced stages. It would be worthwhile to integrate genetic variability in vitamin $D$ metabolism genes as well as biomarkers of infection and inflammation in future studies of vitamin D and lymphoma. Moreover, pooled analyses of large data sets are certainly needed to investigate, with sufficient statistical pool may be required to establish the relation between vitamin D concentrations and individual lymphoma subtypes.

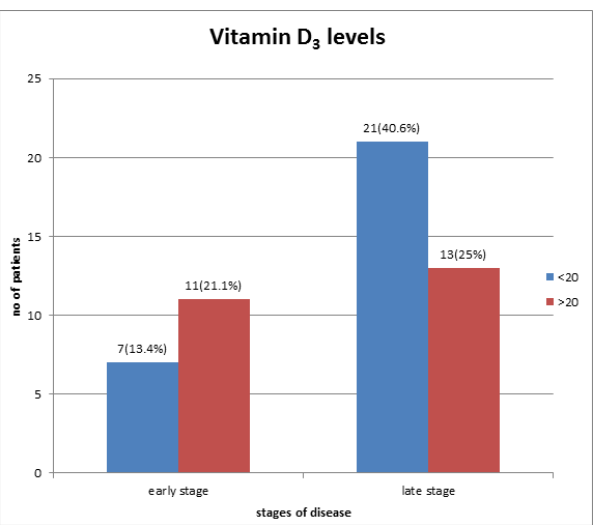

Figure.1. Decreased Levels Of Vitamin $\mathrm{D}_{3}$ In The Advanced Stages Of Disease

Characteristics Of Patients With Interim Treatment Response

\begin{tabular}{|c|l|l|l|}
\hline Features & $\begin{array}{l}\text { Number of } \\
\text { patients in } \\
\text { complete } \\
\text { remission N(\%) }\end{array}$ & $\begin{array}{l}\text { Number of patients } \\
\text { not in complete } \\
\text { remission N(\%) }\end{array}$ & value \\
\hline Vitamin $\mathrm{D}_{3}$ & & & \\
\hline$<20$ & $21(53.8 \%)$ & $5(55.5 \%)$ & 0.983 \\
\hline$>20$ & $18(46 \%)$ & $4(44.4 \%)$ & \\
\hline
\end{tabular}


Table No 1. Univariate Analysis Of Interim Treatment Response With Various Prognostic Variables Shows No Significant Correlation ( P Value $<0.05$ )

Characteristics Of Patients With End Of Treatment Response

\begin{tabular}{|l|l|l|l|}
\hline Features & $\begin{array}{l}\text { Number of } \\
\text { patients in } \\
\text { complete } \\
\text { remission N(\%) }\end{array}$ & $\begin{array}{l}\text { Number of } \\
\text { patients not in } \\
\text { complete } \\
\text { remission N(\%) }\end{array}$ & P value \\
\hline Vitamin $\mathrm{D}_{3}$ & & & \\
\hline$<20$ & $25(52 \%)$ & $3(75 \%)$ & 0.377 \\
\hline$>20$ & $23(47.9 \%)$ & $1(25 \%)$ & \\
\hline
\end{tabular}

Table No 2. Univariate Analysis Of End Of Treatment Response With Various Prognostic Variables

\section{REFERENCES}

1. Rugbjerg K, Maraldo M, Aznar MC, Cutter DJ, Darby SC, Specht L, et al. Long-term hospitalisation rates among 5-year survivors of Hodgkin lymphoma in adolescence or young adulthood: A nationwide cohort study. International journal of cancer. 2017;140(10):2232-45.

2. Ph D, Chan WC, Gascoyne RD. NIH Public Access. 2010;362(10):875-85.

3. Plasma 25-hydroxyvitamin D concentration and lymphoma risk: results of the European Prospective Investigation into Cancer and Nutritionl-3Anna Euczynska, Rudolf Kaaks et al 2013

4. Vardhana S, Younes A. The immune microenvironment in Hodgkin lymphoma: T cells, B cells, and immune checkpoints. Haematologica. 2016;101(7):794-802.

5. Mark A, Hajdu M, Varadi Z, Sticz TB, Nagy N, Csomor J, et al. Characteristic mTOR activity in Hodgkin-lymphomas offers a potential therapeutic target in high risk disease-a combined tissue microarray, in vitro and in vivo study. BMC cancer. 2013;13(1):250.

6. Season of diagnosis is a prognostic factor in Hodgkin's lymphoma: a possible role of sun-induced vitamin D AC Porojnicu ${ }^{*}, 1,2$, TE Robsahm3, AH Ree4 and J Moanl,5 1Department of Radiation Biology, Institute for Cancer Research, Montebello, 0310 Oslo, Norway; 2Department of Biophysics and Cell Biotechnology, Carol Davila University of Medicine and Pharmacy, 15-205. 